\title{
Granulomatöse Bronchiolitis nach Aspiration von Lycopodium-Sporen bei einem Feuerspucker
}

\author{
Granulomatous Bronchiolitis due to Aspiration of Lycopodium Spores \\ by Fire-Breathing
}

Autoren

Institute

\section{A. Morresi-Hauf' , A. Neher ${ }^{2}$, W. Wöckel ${ }^{3}$, H. Kammler-Baumann ${ }^{4}$}

${ }^{1}$ Institut für Pathologie, Asklepios Fachkliniken München-Gauting (Leiterin: Dr. Alicia T. Morresi-Hauf)

2 Klinik für Pneumologie, Asklepios Fachkliniken München-Gauting (Chefarzt: Prof. Dr. Karl Häußinger)

3 Ehemals Institut für Pathologie, Asklepios Fachkliniken München-Gauting (Leiterin: Dr. Alicia T. Morresi-Hauf)

${ }^{4}$ Lungenfacharztpraxis, München eingereicht 12.6.2008

akzeptiert 21.7.2008

Bibliografie

DOI $10.1055 / \mathrm{s}-2008-1038256$

Online-Publikation: 5.9.2008

Pneumologie 2009; 63: 67-71

(c) Georg Thieme Verlag KG

Stuttgart · New York

ISSN 0934-8387

Korrespondenzadresse

Dr. Alicia T. Morresi-Hauf

Institut für Pathologie,

Asklepios Fachkliniken

München-Gauting

Robert Koch-Allee 2

82131 Gauting

a.morresi-hauf@asklepios.com

\section{Zusammenfassung \\ $\nabla$}

Hintergrund: Lungenerkrankungen als Folge einer Aspiration stellen häufig einen unerwarteten Befund und somit eine diagnostische Herausforderung dar.

Kasuistik: Wir berichten über einen 23-jährigen Patienten mit Husten, Fieber und Lungeninfiltraten. Aufgrund der histologisch nachgewiesen granulomatösen Lungenentzündung wurde zuerst eine Tuberkulose angenommen. Bei der histologischen Bearbeitung der operativ gewonnenen Lungengewebsproben wurde eine bronchiolozentrische z.T. nekrotisierende epitheloidzellgranulomatöse Entzündung mit Nachweis von fremden Partikeln im Sinne einer Aspirationsbronchiolitis festgestellt.

Ergebnisse: Nur die wiederholte gezielte anamnestische Erhebung ermöglichte die Identifikation der Noxe. Die fremden Partikel entsprachen Lycopodium-Sporen, die der Patient bei gelegentlichem „Feuerspucken“ aspiriert hatte.

Schlussfolgerungen: Das ist der erste Bericht über eine Lungenerkrankung nach Aspiration von Lycopodium-Sporen bei einem Feuerspucker. Er zeigt, dass bei Aspirationen die gezielte Befragung so entscheidend wie der histologische Befund zur endgültige Abklärung des Krankheitsbildes ist.

\section{Einleitung \\ $\nabla$}

Außerhalb der typischen klinischen Konstellation einer Aspirationspneumonie bzw. bei gesunden Patienten kann der histologische Nachweis granulomatöser Veränderungen mit Fremdkörpern einen schwer zu interpretierenden Befund darstellen. Wir berichten einen Fall einer Aspirationsbronchiolitis bei einem jungen Mann, der sich gelegentlich als „Feuerspucker“ unter Einsatz von Lycopodium-Sporen betätigte.

\section{Abstract \\ $\nabla$}

Background: Lung disease due to aspiration is often unsuspected and represents a diagnostic challenge.

Case Report: We report on a 23-year-old male patient with cough, fever and infiltrates on the chest radiograph. The lung biopsy showed granulomatous changes consistent with tuberculosis. The surgical lung biopsy revealed aspiration bronchiolitis with necrosis, granulomatous changes and particulate foreign material.

Results: The aspirated matter could be identified only after repeated interrogation specifically directed to the matter. The patient had aspirated Lycopodium spores in the course of occasional fire-breathing.

Conclusions: This is the first report on pulmonary disease due to aspiration of Lycopodium spores in the course of fire-breathing. It shows that the anamnesis is as crucial as histology for the definitive diagnosis of aspiration disease. 


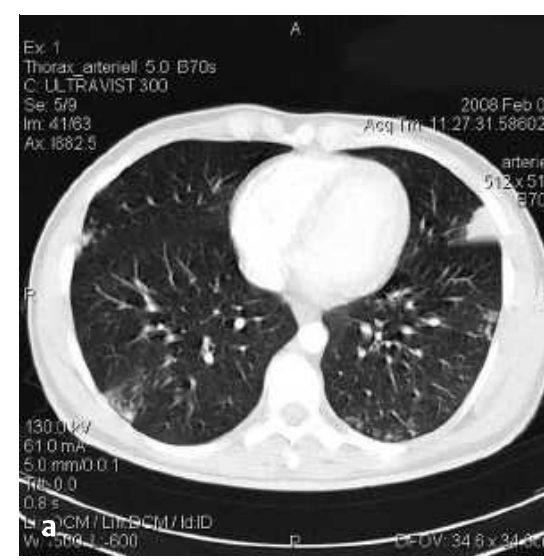

Abb. 1 Thorax-CT: a Diffus verteilte periphere herdförmige fleckig-konfluierende Infiltrate beiderseits. b Betonung der Infiltrate in den Unterfeldern und thoraxwandnah.

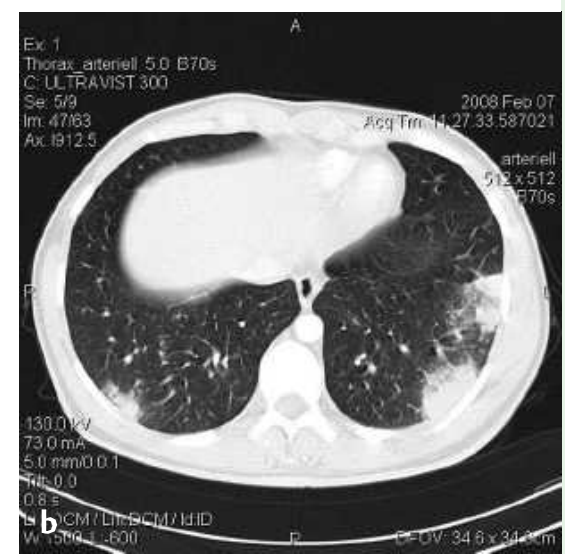

(s. Abb.1). Eine Antibiotikatherapie brachte weder radiologisch noch klinisch eine Besserung.

Bei weiterbestehenden Hustenanfällen, Nachtschweiß und Gewichtsverlust von insgesamt $4 \mathrm{~kg}$ wurde der Patient zur Abklärung der intrapulmonalen Infiltrate im Februar 2008 stationär aufgenommen.

Anamnestisch ließ sich ein allergisches Asthma eruieren, das seit einigen Jahren weitgehend asymptomatisch ist. Der Patient ist Raucher, ist beschäftigt als Barkeeper und berichtete über Schimmelpilze an den Wänden des Arbeitsplatzes.

Laborchemisch fand sich ein erhöhtes CRP von 46,5 mg/l (Norm bis 3), eine Leukozytose mit 18,9 Giga/l, Fibrinogen $>999 \mathrm{mg} / \mathrm{dl}$, in der Proteinelektrophorese eine alpha-2-Globulinerhöhung auf 17,4 rel.\%. Die übrigen routinemäßig erfassten Laborparameter einschließlich Procalcitonin waren normal. Rheumafaktor, p-ANCA und c-ANCA, Autoimmundiagnostik waren unauffällig. Der HIV-Test war negativ.

In der Allergologiediagnostik fand sich ein stark erhöhtes IGE mit $2.488 \mathrm{kU} / \mathrm{l}$, RAST-Klasse 6 für Lieschgras und Klasse 5 für Birke.

Mikrobiologisch fanden sich keine pathogenen Keime im Sputum. Der Tuberkulintest fiel negativ aus.

Im bronchoskopisch gewonnenen Lungenbiopsat fand sich eine nicht nekrotisierende granulomatöse Entzündung, ohne fluoreszenzmikroskopischen Nachweis säurefester Bakterien.

Bei weiterhin unklarem Befund wurden 2 Exzisate vom rechten Lungenunterlappen in MIC-Technik gewonnen: Diese zeigten mikroskopisch eine z.T. nekrotisierende granulomatöse Entzündung vor allem in Bereich der Bronchiolen, wiederum ohne fluoreszenzmikroskopischen Nachweis säurefester Bakterien.

Da der morphologische Befund trotzdem mit einer Tuberkulose vereinbar war, wurde eine antituberkulöse Therapie eingeleitet. In der weiteren histologischen Bearbeitung fanden sich aber Par- tikel Fremdmaterials in den Granulomen. Daraufhin wurde die Diagnose einer granulomatösen Bronchiolitis bei Fremdkörperaspiration gestellt (s. Kasten: „morphologische Befunde“).

Trotz intensiver Befragung konnten weder inhalative Noxen noch Hinweise auf eine Aspiration, auf Mikroaspirationen oder auf eine Refluxkrankheit in Erfahrung gebracht werden. Da die Fremdkörperpartikel z.T. eine starke Ähnlichkeit mit Eiern von Paragonimus oder Schistosoma boten, wurde trotz des Fehlens einer eosinophilen entzündlichen Komponente eine parasitäre Erkrankung erörtert, die sich wiederum nicht bestätigen ließ.

Erst nach Entlassung und nach nochmaliger gezielter Anamnese durch die niedergelassene Pneumologin eröffnete der Patient, dass er sich gelegentlich als „Feuerspucker“ betätigte und dies unter Einsatz von Lycopodium-Sporen. Dieses Material wurde über Internet bestellt und histologisch bearbeitet: mikroskopisch zeigten diese Sporen einen identischen Befund wie die Fremdkörperpartikel im Biopsat des Patienten. Somit konnte festgestellt werden, dass die Lungenveränderungen eine fremdkörpergranulomatöse Bronchiolitis nach Aspiration von Lycopodium-Sporen darstellte. Die antituberkulöse Therapie wurde nach insgesamt 4 Wochen beendet.

Nach Noxen-Karenz und ohne weitere medikamentöse Behandlung besteht eine klinische Besserung mit Rückbildung von Husten und Fieber.

\section{Morphologische Befunde}

- Im transbronchialen Lungenbiopsat fanden sich nicht nekrotisierende Epitheloidzellgranulome mit gelegentlichen mehrkernigen Riesenzellen.

- Die operativ gewonnenen Lungenexzisate zeigten histologisch wiederum eine epitheloidzellgranulomatöse Entzündung, hier allerdings stärker ausgeprägt und mit herdförmigen Nekrosen (s. Abb. 2 u. 3). Die entzündlichen Veränderungen trafen vorwiegend die Wände der Bronchiolen sowie das peribronchioläre Gewebe mit Granulomen mit mehrkernigen Riesenzellen mit intrazytoplasmatischen Einschlüssen, die in der Routine-Färbung Schaumann-Körperchen ähnelten bzw. als solche interpretiert werden könnten (s. Abb.4). Diese Einschlüsse waren unter der Polarisationsoptik schwach doppelbrechend und zeigten sich in der Grocott-Färbung als ovale oder dreieckige Partikel von 20 - 30 $\mu \mathrm{m}$ Durchmesser, umgeben von kurzen Stacheln. In einzelnen Partikeln waren kleine eosinophile Granula bzw. Spherula oder Kügelchen zu sehen (s. $\odot$ Abb.5). Die über Internet bestellten „Lycopodium-Sporen zum Feuerspucken“ zeigten nach histologischer Bearbeitung einen identischen mikroskopischen Befund (s. Abb.6).

\section{Diskussion \\ $\nabla$}

„Aspirationspneumonien“ und „Aspirationsbronchiolitis" mit Nachweis von Fremdkörperpartikeln Fremdkörperpartikel in der Lunge sind im Rahmen von Aspirationspneumonien bei Zustand nach akzidenteller Aspiration zu finden.

Ferner finden sich fremde Partikel in der Lunge bei Pneumokoniosen, Lipidpneumonien oder innerhalb der pulmonalen Blutgefäße oder perivaskulär bei Zustand nach intravenöser Applikation - z.B. bei Drogensüchtigen [1]. Diese letzten Situationen werden aber hier wegen des sehr charakteristischen klinischen 

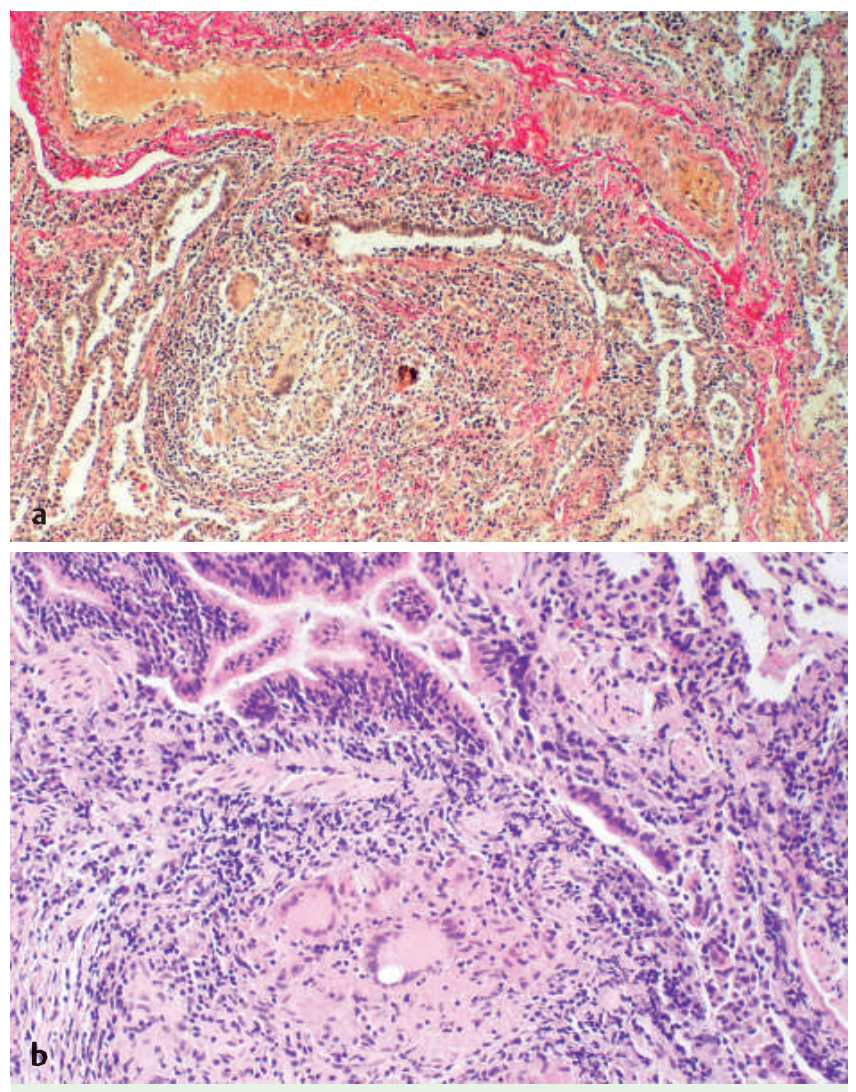

Abb. 2 Bronchiolozentrische epitheloidzellgranulomatöse Entzündung (a Elastika-van-Gieson-Färbung 25×, b Hämatoxylin-Eosin-Färbung 50×).

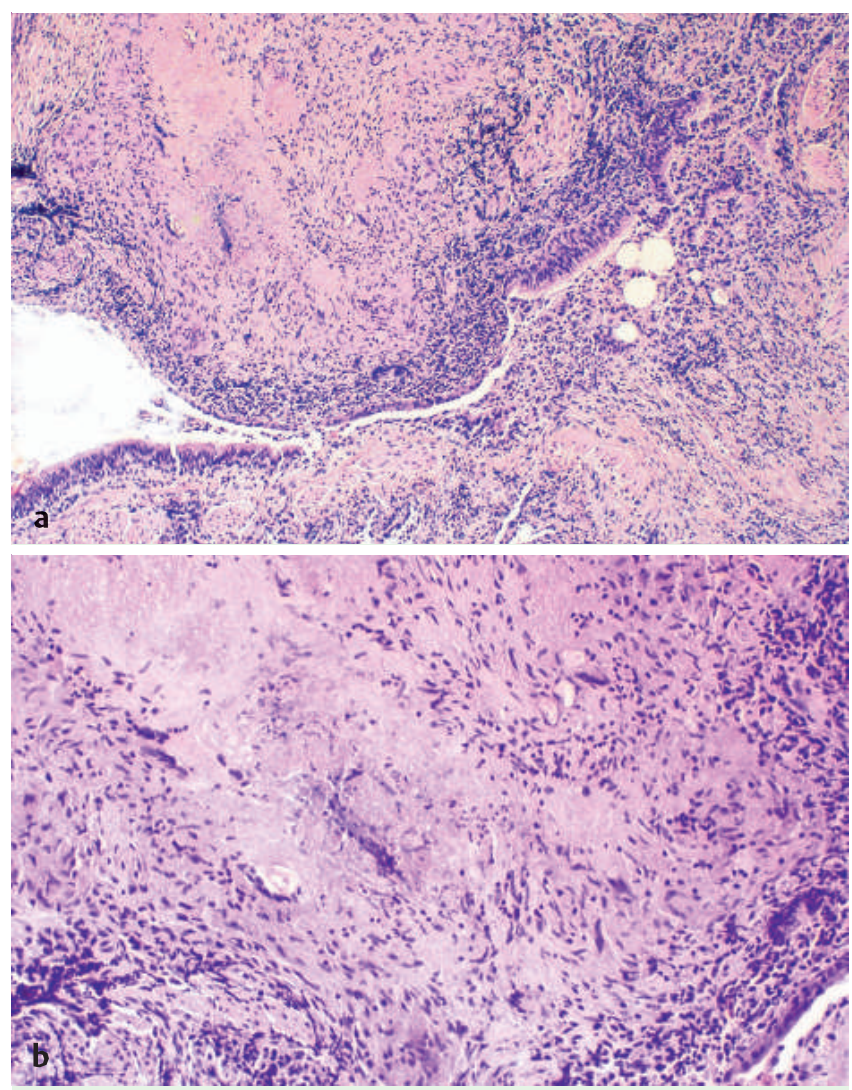

Abb.3 Zum Teil nekrotisierende Epitheloidzellgranulome peribronchiolär (a Hämatoxylin-Eosin-Färbung 25×, b Hämatoxylin-Eosin-Färbung 50×).
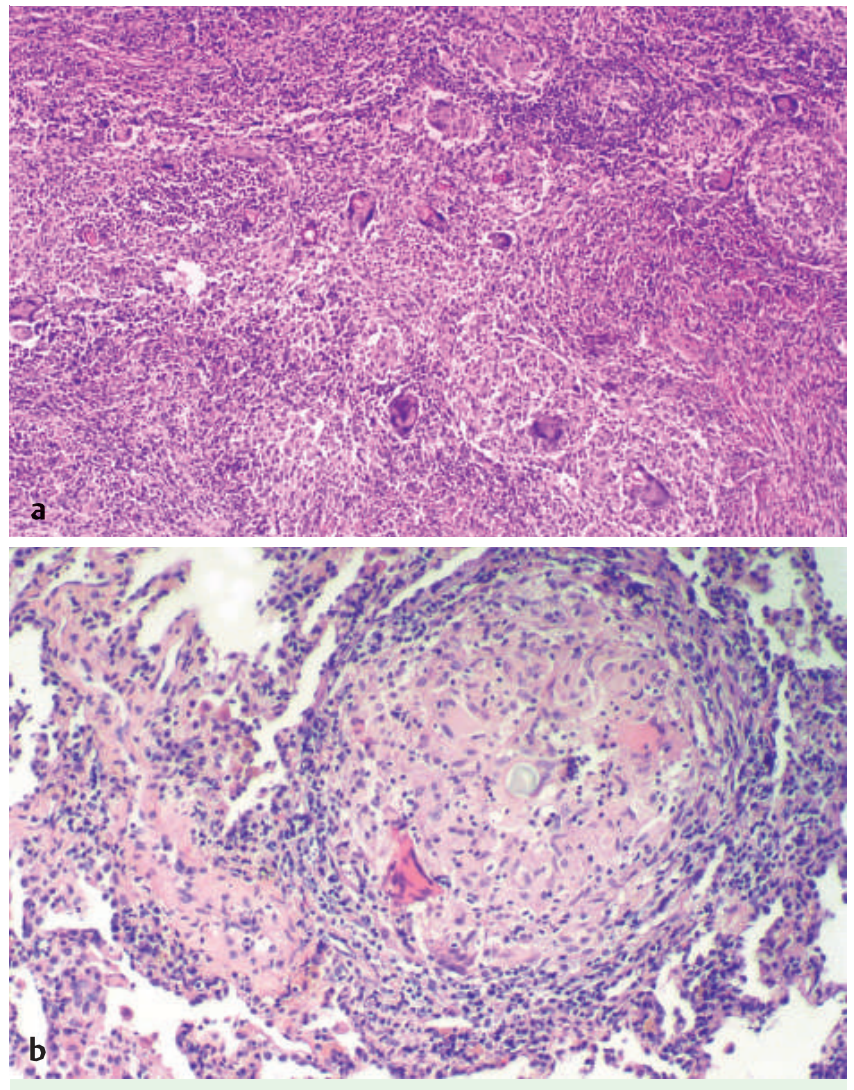

Abb.4 Epitheloidzellgranulome mit Partikeln Fremdmaterials im Zytoplasma von Riesenzellen (a PAS-Färbung 25×, b Hämatoxylin-Eosin-Färbung $50 \times)$.
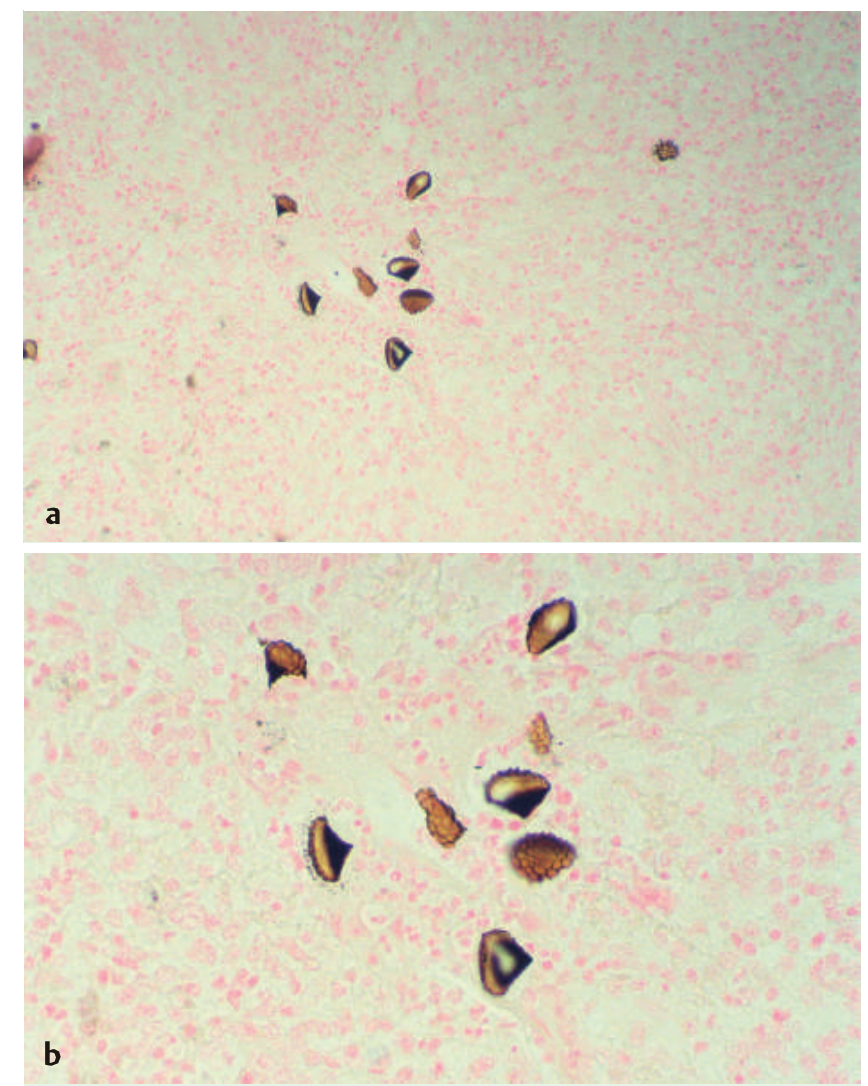

Abb.5 Floride Entzündung im Lungengewebe mit ähnlichen z. T. etwa dreieckigen Partikeln Fremdmaterials (a Grocott-Färbung 50×, b GrocottFärbung $100 \times$ ). 

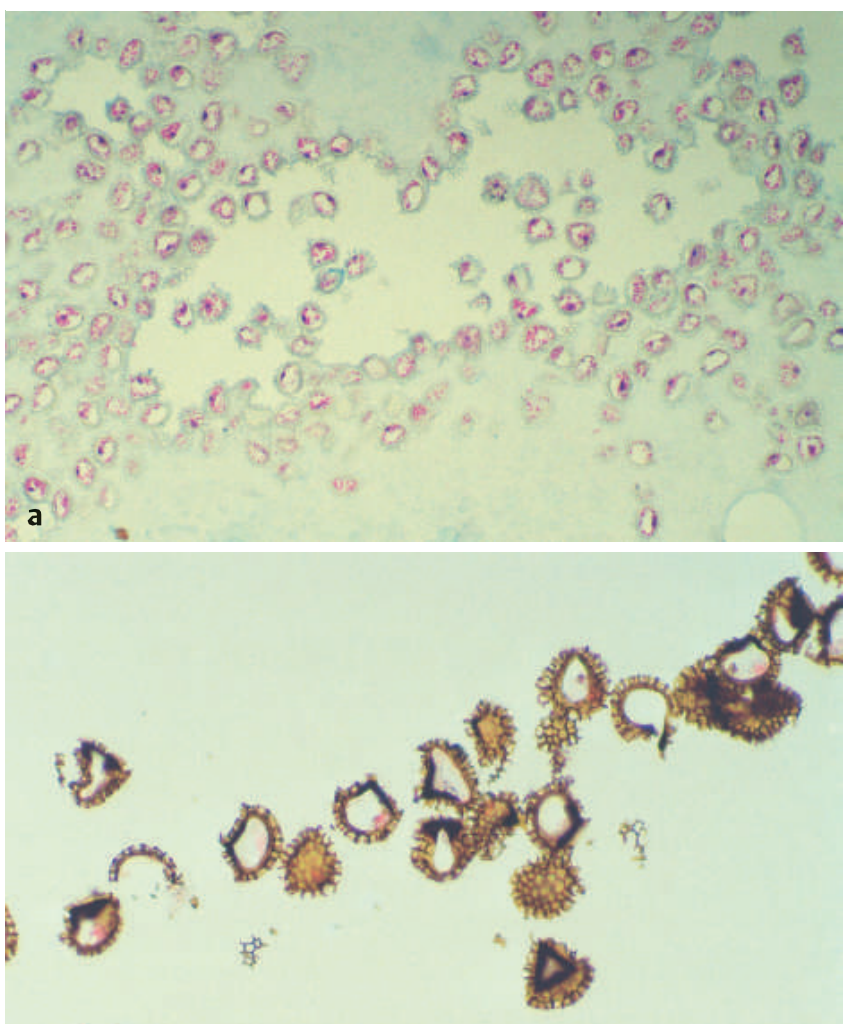

b

Abb. 6 Über Internet bestellte „Lycopodium-Sporen zum Feuerspucken“ nach Einbettung in Paraffin und Grocott-Färbung mit identischem mikroskopischem Bild (a Hämatoxylin-Eosin-Färbung 50×, b Grocott-Färbung $100 \times)$.

und/oder pathologischen Befundes nicht weiter behandelt bzw. berücksichtigt.

„Aspirationspneumonien“ bzw. entzündliche Veränderungen der Lunge wegen Aspiration von Speise- oder anderen Partikeln sind ein relativ häufiger Befund bei Obduktionen von älteren geschwächten oder bettlägerigen Patienten. Sie können auch bei Patienten mit Erkrankungen des Ösophagus und/oder Magens oder bei neurologischen bzw. psychiatrischen Krankheiten vorkommen oder auch bei gesunden Patienten nach Episoden von Bewusstlosigkeit oder Beeinträchtigung des Bewusstseins, wie nach Krämpfen, Synkopen oder bei Drogensüchtigen nach Überdosis.

Marik weist auf die relativ hohe Frequenz von durch Aspiration verursachten entzündlichen Lungenkrankheiten bei Bewohnern von Pflegeheimen hin und auf eine für die Behandlung bedeutsame Unterteilung in Aspirationspneumonie als ein infektiöser Prozess als Folge der Inhalation von mit Bakterien kontaminierten oropharyngealen Sekretionen und Aspirationspneumonitis oder Mendelsohn-Syndrom als ein chemischer Schaden als Folge der Aspiration von sterilem Mageninhalt [2].

Auch bei Zustand nach Lungen- bzw. Herz-Lungen-Transplantation können Veränderungen im Sinne einer Aspirationsbronchiolitis auftreten [3].

Klinisch sind Dyspnoe, Fieber und Husten die häufigsten Beschwerden.

Im Röntgenbild wurden diffuse kleinknotige Infiltrationen als charakteristisch angesehen [4]. Bilaterale Infiltrate oder Knoten, aber auch unilaterale Infiltrate und einzelne Knoten sind be- schrieben worden [1]. In Fällen mit prädominanter bronchiolitischer Komponente zeigt das Röntgenbild hauptsächlich zentrilobuläre Knötchen [5].

Die histologische Diagnose wird durch den Nachweis der Fremdpartikel im Zusammenhang mit entzündlichen Veränderungen des Lungengewebes gestellt. Diese letzten zeigen das Bild einer Pneumonie oder einer organisierenden Pneumonie („BOOP“) und nicht selten das Bild einer Bronchiolitis mit multifokaler bronchiolozentrischer granulomatöser Entzündung mit Fremdkörperreaktion. Diese diffuse „Aspirationsbronchiolitis“ ist vor allem bei alten Patienten mit Dysphagie und/oder bettlägerig und zum Teil mit neurologischen Krankheiten oder Demenz beschrieben worden [4], aber auch in jüngeren Patienten mit Refluxkrankheit [5]. Sie soll einen schleichenderen Verlauf als die Aspirationspneumonie haben und kann klinisch mit einem spät beginnenden Asthma verwechselt werden. Sie dürfte die Folge von wiederholten Mikroaspirationen sein. In einer Autopsiestudie handelte es sich bei den Fremdpartikeln hauptsächlich um Speisereste aus dem Mageninhalt [4].

Außerhalb der typischen klinischen Konstellation einer Aspiration stellt der Nachweis von Fremdkörperpartikeln in Biopsaten, Exzisaten oder Resektaten häufig einen unerwarteten Befund dar. Dabei findet sich vor allem das mikroskopische Bild einer BOOP (Bronchiolitis obliterans mit organisierender Pneumonie), häufig in Kombination mit Veränderungen im Sinne einer Bronchopneumonie oder einer Bronchiolitis und Granulomen, vor allem assoziiert mit Partikeln von Fremdmaterial [1,6]. Seltener findet sich keine organisierende Pneumonie, sondern nur fibrotische Veränderungen oder eine abszedierende Entzündung im Zusammenhang mit dem Nachweis von Fremdmaterial [1].

In der morphologischen Differenzialdiagnose könnten eine BOOP oder Entitäten, die mit granulomatösen Veränderungen einhergehen, wie eine bronchozentrische Granulomatose, eine exogen-allergische Alveolitis, eine Sarkoidose oder ein Morbus Wegener erörtert werden. Die strikte Berücksichtigung der morphologischen Diagnosekriterien dieser Entitäten, aber vor allem der Nachweis von Fremdmaterial im Zusammenhang mit den granulomatösen Veränderungen führen in der Regel zur korrekten Diagnose.

\section{Die Fremdpartikel}

Unter den Fremdpartikeln finden sich am häufigsten Anteile pflanzlichen Gewebes, vor allem bei Aspiration von Speiseresten bzw. bei Zustand nach Reflux und Aspiration [4].

Ferner sind Anteile quergestreifter Muskulatur aus Speiseresten sowie Talkum, Zellulose, Crospovidon und Kayexalat beschrieben worden [1]. Talkum, Zellulose und Crospovidon werden für die Herstellung von Arzneimitteln benutzt, so dass deren Nachweis in Aspirationspneumonien wahrscheinlich auf die akzidentelle Aspiration dieser Medikamente zurückzuführen ist. Sie sind auch in den pulmonalen Arterien und im periarteriellen Interstitium bei Drogensüchtigen mit intravenöser Substanzzufuhr beschrieben worden. Kayexalat wird bei der Behandlung von Hyperkaliämie benutzt.

Als eine Kuriosität ist die akzidentelle Aspiration von Psyllium, ein pflanzliches, als Ballaststoff für die Behandlung von Obstipation benutztes Präparat, anzusehen [6].

\section{Lycopodium}

In der Literatur fanden wir nur eine Mitteilung, wobei Fremdpartikel in Corpora amylacea in der Lunge als aspirierte Lycopodium-Sporen interpretiert wurden [7]. Über Lycopodium-Sporen 
als Noxe einer Aspirationsbronchiolitis ist bis jetzt in der Literatur nicht berichtet worden.

Lycopodium oder Bärlappe sind farnartige Pflanzen, deren Sporen (Lycopodiumstaub) früher in der Industrie bei der Herstellung von Arzneimitteln (als Bestandteil von Pillen und Suppositorien), in der Homöopathie, in der Kosmetik-Industrie und als Puder für Handschuhe verwendet wurden. Einzelne Publikationen berichten über Lycopodium-Granulome in der Haut und im Bereich von chirurgischen Verletzungen nach Benutzung von mit Lycopodium-Sporen behandelten Handschuhen [8,9].

Heutzutage werden Lycopodium-Sporen weiterhin bei der Herstellung bzw. Verpackung von Kondomen verwendet. Sie sind als Noxe von Asthma und allergischen Beschwerden bei Mitarbeitern von Kondomfabriken beschrieben worden [10,11]. Auch bei der Abklärung von Sexualstraftaten wird der Nachweis von Lycopodium-Sporen nach Vergewaltigung in der forensischen Medizin eingesetzt [12]. Als Kuriosität ist der Nachweis von Lycopodium-Sporen in Prostatabiopsaten anzusehen, bei Zustand nach transrektaler bioptischer Gewebsentnahme unter Einsatz von mit Kondomen geschützten Geräten [13].

Ferner sind Lycopodium-Sporen seit dem Mittelalter in der Erzeugung pyrotechnischer Effekte benutzt worden und werden heutzutage noch von Magiern und im Showbereich verwendet. Anleitungen für das Erzielen von attraktiven Feuereffekten mit diesem Stoff, darunter auch fürs „Feuerspucken“, sind u. a. im Internet zu finden.

Der fehlende klinische Hintergrund bei unserem Patienten (junges Alter, keine neurologische oder ösophago-gastrale Erkrankung, fehlende eindeutige anamnestische Hinweise auf eine akzidentelle Aspiration) erschwerten hier die Interpretation der granulomatösen Veränderungen als Folge einer Aspiration. Wegen der Morphologie der fremden Partikel wurde eine parasitäre Erkrankung mit Paragonimus oder Schistosoma erwogen, wobei bereits auf die Ähnlichkeit der Lycopodium-Sporen mit den Eiern dieser Erreger in Fremdkörpergranulomen in der Haut hingewiesen worden ist [8].

Die nur zögerliche Preisgabe der Information über seine gelegentliche Betätigung als „Feuerspucker“ seitens des Patienten, führte im vorliegenden Fall zu der relativ späten Identifizierung der Noxe. Nur die enge Kooperation aller mit diesem Fall beschäftigten Ärzte führte letztendlich zur Abklärung des Befundes mit der hier vorliegenden ersten Beschreibung einer Aspirationsbronchiolitis mit Lycopodium-Sporen bei einem Feuerspucker.

\section{Literatur}

1 Mukhopadhyay S, Katzenstein AL. Pulmonary disease due to aspiration of food and other particulate matter: a clinicopathologic study of 59 cases diagnosed on biopsy or resection specimens. Am J Surg Pathol 2007; 31: $752-759$

2 Marik PE. Aspiration pneumonitis and aspiration pneumonia. N Engl J Med 2001; 344: 665-671

3 Miyagawa-Hayashino A, Wain JC, Mark EJ. Lung transplantation biopsy specimens with bronchiolitis obliterans or bronchiolitis obliterans organizing pneumonia due to aspiration. Arch Pathol Lab Med 2005; 129: $223-226$

4 Matsuse T, Oka T, Kida K et al. Importance of diffuse aspiration bronchiolitis caused by chronic occult aspiration in the elderly. Chest 1996; 110: $1289-1293$

5 Barnes TW, Vassallo R, Tazelaar HD et al. Diffuse bronchiolar disease due to chronic occult aspiration. Mayo Clin Proc 2006; 81: 172 - 176

6 Janoski MM, Raymond GS, Puttagunta L et al. Psyllium aspiration causing bronchiolitis: radiographic, high-resolution $\mathrm{CT}$, and pathologic findings. AJR Am J Roentgenol 2000; 174: 799-801

7 Hollander DH, Hutchins GM. Central spherules in pulmonary corpora amylacea. Arch Pathol Lab Med 1978; 102: 629-630

8 Del Rosario RN, Barr RJ, Graham BS et al. Exogenous and endogenous cutaneous anomalies and curiosities. Am J Dermatopathol 2005; 27 : $259-267$

9 Nadjem MA, Graham JH, Johnson FB. Lycopodium granuloma. J Cutan Pathol 1988; 15: 120-123

10 Cullinan P, Cannon J, Sheril D et al. Asthma following occupational exposure to Lycopodium clavatum in condom manufacturers. Thorax 1993; 48: $774-775$

11 Rask-Andersen A, Boman J, Grängsjö A et al. Asthma, skin symptoms, and allergy in a condom factory. Allergy 2000; 55: 836-841

12 Berkefeld $K$. A possibility for verifying condom use in sex offenses. Arch Kriminol 1993; 192: $37-42$

13 Andersen TC, Jürgensen GW, Christensen E. Lycopodium spores in transrectal ultrasound-guided core biopsies of the prostate. Scand J Urol Nephrol 1998; 32: $148-149$ 